If we now let $\psi^{*}=r^{* 3} \psi^{\prime}$, this equation becomes

$$
\gamma^{j}\left(\frac{\partial}{\partial x^{j *}}-\frac{e}{c} \frac{i}{h} \phi_{j}^{*}\right) \psi^{\prime}=-\mu r^{2} \psi^{\prime} .
$$

The factor $-r^{2}$ on the right-hand side of this equation shows that the Dirac equation for an electron is not invariant under inversions. However, if we set $\phi_{j}=0$ and $\mu=0$, then equation (15) is numerically invariant under inversions. This is done in the neutrino theory of light. Hence that theory has the same invariance properties as the Maxwell theory. Veblen $\dagger$ and Dirac $\ddagger$ have both shown that there is no nonsingular analog of the Dirac equation which is conformally invariant. The result given here shows how the invariance fails. We have given the detailed treatment of the behavior of the Dirac equation under the four-dimensional inversion; the three-dimensional inversion may be treated by restricting the range of indices in (7), (8), and (12) to $1,2,3$ and adding the equation $x^{4 *}=x^{4}$ to (7).

UNIVERSITY OF WASHINGTON

\title{
NOTE ON DEGREE OF TRIGONOMETRIC AND POLYNOMIAL APPROXIMATION TO AN ANALYTIC FUNCTION $\$$
}

\section{J. L. WALSH AND W. E. SEWELL}

1. Introduction. Well known results $\|$ relate the continuity properties of a real function $f(x)$ to the degree of approximation to $f(x)$ by trigonometric sums and by polynomials in $x$. In more recent years further results $\uparrow$ have related the continuity properties of a complex function $f(z)$ to the degree of approximation to $f(z)$ by polynomials in the complex variable $z$. The object of the present note is to obtain some new results lying on the border line of these two general fields of research.

To be more explicit, if $f(z)$ is analytic in the annulus $\rho>|z|>1 / \rho<1$, the degree of convergence on $|z|=1$ of the Laurent development of

$\dagger \mathrm{O}$. Veblen, $A$ conformal wave equation, Proceedings of the National Academy of Sciences, vol. 21 (1935), p. 484.

$\ddagger$ P. A. M. Dirac, Wave equations in conformal space, Annals of Mathematics, (2), vol. 37 (1936), p. 429.

$\S$ Presented to the Society, September 6, 1938.

|| Due especially to S. Bernstein, Jackson, Lebesgue, Montel, and de la Vallée Poussin.

I Due especially to J. Curtiss, Sewell, and Walsh. 
$f(z)$ is intimately connected with the continuity properties of $f(z)$ on the two circles $|z|=\rho$ and $|z|=1 / \rho$. This fact is already known (Bernstein, de la Vallée Poussin), if $f(z)$ has poles or certain other singularities on those two circles, and is established in the present note if $f(z)$ or one of its derivatives satisfies a Lipschitz condition on $|z|=\rho$ and $|z|=1 / \rho$.

Once the latter connection is established, standard methods involving suitable conformal transformations enable us to study the relation between polynomial approximation to an analytic function $f(z)$ on the segment $-1 \leqq z \leqq 1$ and the continuity properties of $f(z)$ on the largest ellipse whose foci are +1 and -1 within which $f(z)$ is analytic. We also make application to the relation between trigonometric approximation on $y=0$ (with $z=x+i y$ ) to an analytic function $f(z)$ with period $2 \pi$ and the continuity properties of the function on the lines $y= \pm b$ bounding a region within which $f(z)$ is analytic.

2. Approximation on the unit circle. We prove the following theorem:

Theorem 1. Let $f(\theta)$ be periodic with period $2 \pi$, and suppose the numbers $a_{n k}$ and $b_{n k}$ (not necessarily real) are given so that

$$
s_{n}(\theta)=\frac{a_{n 0}}{2}+\sum_{k=1}^{n}\left(a_{n k} \cos k \theta+b_{n k} \sin k \theta\right),
$$

with the relation, for $n=1,2, \cdots$ and for all $\theta$,

$$
\left|f(\theta)-s_{n}(\theta)\right| \leqq M / n^{p+\alpha+1} \rho^{n}, \quad 0<\alpha \leqq 1, \rho>1,
$$

where $p$ is a non-negative integer and $M$ is a constant. Then the function

$$
\begin{aligned}
F(z) & \equiv \lim _{n \rightarrow \infty}\left[c_{n 0}+\sum_{k=1}^{n}\left(c_{n,-k} z^{-k}+c_{n k} z^{k}\right)\right], \\
2 c_{n k} & =a_{n k}-i b_{n k}, \quad 2 c_{n,-k}=a_{n k}+i b_{n k},
\end{aligned}
$$

coincides with $f(\theta)$ on the circle $|z|=1$, with $z=\cos \theta+i \sin \theta$, and $F(z)$ is analytic in the annulus $\rho>|z|>1 / \rho$ and continuous in the corresponding closed region. For $z_{1}$ and $z_{2}$ on $|z|=\rho$ or $1 / \rho$ we have*

$$
\left|F^{(p)}\left(z_{1}\right)-F^{(p)}\left(z_{2}\right)\right| \leqq L \cdot\left|z_{1}-z_{2}\right|^{\alpha} \cdot|\log | z_{1}-z_{2}||^{\beta},
$$

where $\beta=0$ if $\alpha<1$, and $\beta=1$ if $\alpha=1$, and where $L$ is a constant independent of $z_{1}$ and $z_{2}$.

* The notation $F^{(p)}(z)$ indicates the pth derivative of $F(z)$, if $p>0$, and the function $F(z)$ itself for $p=0$. Here and below, such derivatives on the boundaries of regions of analyticity are considered in the one-dimensional sense. 
Inequality (1) written for successive indices implies

$$
\left|s_{n+1}(\theta)-s_{n}(\theta)\right| \leqq 2 M / n^{p+\alpha+1} \rho^{n}
$$

for all $\theta$, an inequality which we write in the form

$$
\left|p_{n+1}(z)-p_{n}(z)\right| \leqq 2 M / n^{p+\alpha+1} \rho^{n}, \quad \text { for }|z|=1,
$$

where $p_{n}(z)$ is the expression in square brackets in (2), a polynomial in $z$ and $1 / z$ of degree $n$, equal to $s_{n}(\theta)$ on $|z|=1$. An easily proved lemma* then yields

$$
\left|p_{n+1}(z)-p_{n}(z)\right| \leqq 2 M \rho / n^{p+\alpha+1}, \quad \text { for } \rho \geqq|z| \geqq 1 / \rho .
$$

In particular, on the two circles $|z|=\rho$ and $|z|=1 / \rho$ we may write

$$
\left|F(z)-p_{n}(z)\right| \leqq 2 M \rho\left[\frac{1}{n^{p+\alpha+1}}+\frac{1}{(n+1)^{p+\alpha+1}}+\cdots\right]<M_{1} / n^{p+\alpha} .
$$

The function $p_{n}(z)$ (not necessarily real) is, on $|z|=\rho$ and on $|z|=1 / \rho$, a trigonometric polynomial in $\theta$ of order $n$ with $z=\rho e^{i \theta}$ or $z=\rho^{-1} e^{i \theta}$, so that by the results of de la Vallee Poussin $\dagger$ the function $F(z)$ satisfies on $|z|=\rho$ and $|z|=1 / \rho$ a condition with respect to $\theta$ of form (3); hence (3) itself is fulfilled.

It is a corollary to our proof of Theorem 1 that the sequence $s_{n}(\theta)$, if defined off the circumference $|z|=1$ as the function $p_{n}(z)$, converges uniformly to $F(z)$ in the closed annulus $\rho \geqq|z| \geqq 1 / \rho$, with an error not greater than $M_{1} / n^{p+\alpha}$.

In the direction of the converse of Theorem 1 we prove the following theorem:

THEOREM 2. Let the function $F(z)$ be analytic in the annular region bounded by the circles $|z|=\rho>1$ and $|z|=1 / \rho$ and continuous in the corresponding closed region, and let $F^{(p)}(z), p$ a non-negative integer, satisfy a Lipschitz condition $\S$ of order $\alpha$ on $|z|=\rho$ and $|z|=1 / \rho$. Let us set

$$
F(z) \equiv \sum_{k=-\infty}^{\infty} c_{k} z^{k}, \quad \rho>|z|>1 / \rho
$$

* Walsh, Interpolation and Approximation by Rational Functions in the Complex Domain, American Mathematical Society Colloquium Publications, vol. 20, New York, 1935, p. 259.

$\dagger$ Leçons sur l'Approximation, Paris, 1919, chap. 4. The results in question are equally valid for real and for complex valued functions.

$\ddagger$ See for instance Walsh and Sewell, this Bulletin, vol. 43 (1937), pp. 557-563.

$\S$ That is to say, let ( 3 ) be satisfied with $\beta=0$. 
then with the notation $a_{k}=c_{k}+c_{-k}, b_{k}=i\left(c_{k}-c_{-k}\right)$, we have on $|z|=1$, with $z=e^{i \theta}$, the relation

$$
\left|F\left(e^{i \theta}\right)-\left[\frac{a_{0}}{2}+\sum_{k=1}^{n}\left(a_{k} \cos k \theta+b_{k} \sin k \theta\right)\right]\right| \leqq M / n^{p+\alpha} \rho^{n},
$$

where $M$ is a constant.

In the usual proof of the validity of the Laurent development (4), it is established that we may write for $\rho>|z|>1 / \rho$ the equation $F(z) \equiv F_{1}(z)+F_{2}(z)$, where

$$
F_{1}(z)=\sum_{k=0}^{\infty} c_{k} z^{k},|z|<\rho ; \quad F_{2}(z)=\sum_{k=-1}^{\infty} c_{k} z^{k},|z|>1 / \rho .
$$

Under the present conditions on $F(z)$, the function $F_{2}(z)$ is analytic on $|z|=\rho$, so that the function $F_{1}^{(p)}(z) \equiv F^{(p)}(z)=F_{2}{ }^{(p)}(z)$ is continuous on $|z|=\rho$ and satisfies on $|z|=\rho$ a Lipschitz condition of order $\alpha$. Similarly the function $F_{2}^{(p)}(z)$ is continuous on $|z|=1 / \rho$ and satisfies on $|z|=1 / \rho$ a Lipschitz condition of order $\alpha$.

By the continuity of $F_{1}(z)$ on $|z|=\rho$ and of $F_{2}(z)$ on $|z|=1 / \rho$, we may write

$$
c_{k}=\frac{1}{2 \pi i} \int_{|t|=\rho} \frac{F_{1}(t) d t}{t^{k+1}}, k \geqq 0 ; \quad c_{k}=\frac{1}{2 \pi i} \int_{|t|=1 / \rho} \frac{F_{2}(t) d t}{t^{k+1}}, k<0 ;
$$

whence for $|z|<\rho$, by the uniform convergence of the series involved, we have

$$
F_{1}(z)-\sum_{k=0}^{n} c_{k} z^{k}=\frac{1}{2 \pi i} \int_{|t|=\rho} F_{1}(t)\left(\sum_{k=n+1}^{\infty} \frac{z^{k}}{t^{k+1}}\right) d t .
$$

If $P_{n}(t)$ is an arbitrary polynomial in $t$ of degree $n$, we have

$$
\int_{|t|=\rho} \frac{P_{n}(t) d t}{t^{k+1}}=0, \quad k>n
$$

so that, for $|z|<\rho,(6)$ may be written

$$
F_{1}(z)-\sum_{k=0}^{n} c_{k} z^{k}=\frac{1}{2 \pi i} \int_{|t|=\rho}\left[F_{1}(t)-P_{n}(t)\right]\left(\sum_{k=n+1}^{\infty} \frac{z^{k}}{t^{k+1}}\right) d t .
$$

A consequence of the Lipschitz condition on $F_{1}^{(p)}(z)$ on $|z|=\rho$ is that there exist* polynomials $P_{n}(t)$ of respective degrees $n$ with

* J. Curtiss, this Bulletin, vol. 42 (1936), pp. 873-878. The proof is based on methods due to Bernstein, Jackson, and de la Vallée Poussin. 


$$
\left|F_{1}(t)-P_{n}(t)\right| \leqq M^{\prime} / n^{p+\alpha},
$$$$
|t|=\rho,
$$

where $M^{\prime}$ is independent of $n$ and of $t$. Thus equation (7) yields

$$
\left|F_{1}(z)-\sum_{k=0}^{n} c_{k} z^{k}\right| \leqq M^{\prime \prime} / n^{p+\alpha} \rho^{n}, \quad|z|=1,
$$

where $M^{\prime \prime}$ is independent of $n$ and of $z$. A similar and similarly proved relation for $F_{2}(z)$ yields the inequality (5) and the theorem.

A remark due to Sewell* concerning degree of convergence of Taylor developments applies to the degree of convergence of the sequence $\sum_{k=0}^{n} c_{k} z^{k}$ to $F_{1}(z)$ on the circle $|z|=\rho$, and also to the degree of convergence of the sequence $\sum_{k=-1}^{-n} c_{k} z^{k}$ to $F_{2}(z)$ on the circle $|z|=1 / \rho$, so that we obtain

$$
\left|F(z)-\sum_{k=-n}^{n} c_{k} z^{k}\right| \leqq\left(M^{\prime \prime \prime} \log n\right) / n^{p+\alpha}, \quad \text { for } \rho \geqq|z| \geqq 1 / \rho,
$$

where $M^{\prime \prime \prime}$ is independent of $n$ and $z$.

It is a matter of indifference whether in Theorem 1 we prove and in Theorem 2 assume that $F^{(p)}(z)$ is continuous merely on the circles $|z|=\rho$ and $|z|=1 / \rho$ or that $F^{(p)}(z)$ is continuous in the closed region $\rho \geqq|z| \geqq 1 / \rho$, for the one condition implies the other. $\dagger$ A similar remark applies to Theorems 3-6.

3. Approximation on the segment $-1 \leqq z \leqq 1$. The analog of Theorem 1 is the following theorem:

THEOREM 3. Let $f(z)$ be defined on the segment $-1 \leqq z \leqq 1$, and let, for $n=1,2, \cdots$, a polynomial $P_{n}(z)$ in $z$ of degree $n$ exist such that on the segment $-1 \leqq z \leqq 1$

$$
\left|f(z)-P_{n}(z)\right| \leqq M / n^{p+\alpha+1} \rho^{n}, \quad \rho>1,0<\alpha \leqq 1,
$$

where $p$ is a non-negative integer. Then the function $f(z)$, if suitably defined, is analytic throughout the interior of the ellipse $\gamma$ whose foci are -1 and +1 and whose semi-sum of axes is $\rho$; moreover $f(z)$ is continuous in the closed interior of $\gamma$ and on $\gamma$ satisfies the condition

$$
\left|f^{(p)}\left(z_{1}\right)-f^{(p)}\left(z_{2}\right)\right| \leqq L \cdot\left|z_{1}-z_{2}\right|^{\alpha} \cdot|\log | z_{1}-z_{2}||^{\beta},
$$

where $\beta=0$ if $\alpha<1$ and $\beta=1$ if $\alpha=1$, and where $L$ is a constant independent of $z_{1}$ and $z_{2}$.

* This Bulletin, vol. 48 (1935), pp. 111-117, Theorem 4.

$\dagger$ This follows by consideration of the functions $F_{1}(z)$ and $F_{2}(z)$ from the results of Walsh and Sewell, loc. cit. 
We map the $z$-plane onto the $w$-plane by the transformation $z=\left(w+w^{-1}\right) / 2$. Under this transformation the image in the w-plane of the segment $-1 \leqq z \leqq 1$ counted twice is the unit circle $|w|=1$, the image in the $w$-plane of $\gamma$ in the $z$-plane counted twice consists of the two circles $|w|=\rho$ and $|w|=1 / \rho$, and the image in the $w$-plane of the interior of $\gamma$ in the $z$-plane counted twice is the annular region $\rho>|w|>1 / \rho$. The polynomial $P_{n}(z)$ corresponds to a polynomial in $w$ and $1 / w$ of degree $n$; that is to say, considered as a function of $w$ it is precisely of the form of the function $p_{n}(z)$ introduced in the proof of Theorem 1, with $c_{n k}=c_{n,-k}$. It follows from Theorem 1 that the transform of $f(z)$ (considered as a function of $w$ ) defined in the annulus as the limit of the sequence $P_{n}(z)$ (considered as a function of $w$ ) satisfies a condition of form (3) with respect to $w$ on the circles $|w|=\rho$ and $|w|=1 / \rho$ and is symmetric in $w$ and $1 / w$. The function $f(z)$, the transform in the $z$-plane of the limit in the $w$-plane of the sequence $P_{n}(z)$, is single-valued interior to $\gamma$, is obviously analytic interior to $\gamma$ except perhaps for $-1 \leqq z \leqq 1$, and is analytic on that segment because continuous there in the two-dimensional sense. By the analyticity of the transformation $z=\left(w+w^{-1}\right) / 2$ on $|w|=\rho$ and $|w|=1 / \rho$, inequality (8) follows, and the proof is complete.

As a corollary to this proof we remark that the sequence $P_{n}(z)$ itself converges uniformly to $f(z)$ on and within $\gamma$, with an error not greater than $M_{1} / n^{p+\alpha}$.

In the direction of the converse of Theorem 3, immediate application of Theorem 2 yields the following theorem:

TheOREM 4. Let $\gamma$ denote the ellipse whose foci are -1 and +1 and whose semi-sum of axes is $\rho$, let the function $f(z)$ be analytic interior to $\gamma$ and continuous in the corresponding closed region, and let $f^{(p)}(z), p$ a non-negative integer, satisfy a Lipschitz condition of order $\alpha,(0<\alpha \leqq 1)$, on $\gamma$. Then for $n=1,2, \cdots$ there exists a polynomial $P_{n}(z)$ of degree $n$ in $z$ such that $\left|f(z)-P_{n}(z)\right| \leqq M / n^{p+\alpha} \rho^{n},(-1 \leqq z \leqq 1)$, where $M$ is a constant independent of $n$ and $z$.

Under the transformation $z=\left(w+w^{-1}\right) / 2$, the function $f(z)$ corresponds to a function of $w$ which is analytic in the neighborhood of $|w|=1$ except perhaps on that circumference, continuous in the twodimensional sense at every point of $|w|=1$, and hence analytic on $|w|=1$ and throughout the annulus $\rho>|w|>1 / \rho$. The function $f\left[\left(w+w^{-1}\right) / 2\right]$ is symmetric in $w$ and $1 / w$, so that the corresponding Laurent polynomials used in the proof of Theorem 2 are in this case symmetric in $w$ and $1 / w$ and hence are polynomials in $z$; Theorem 4 follows from Theorem 2 . 
It is not without interest to notice that the successive approximating Laurent polynomials that here present themselves in the w-plane are of the form $\sum_{k=0}^{n} c_{k}\left(w^{k}+w^{-k}\right)$, where $c_{k}$ is independent of $n$, so that the approximating polynomials $P_{n}(z)$ of Theorem 4 are of the form

$$
\sum_{k=0}^{n} c_{k} T_{k}(z)
$$

where $c_{k}$ is independent of $n$, and where $T_{k}(z)_{1} \equiv w^{k}+w^{-k}$ is a polynomial of the set studied by Tschebycheff: $T_{0}(z)=2, T_{1}(z) \equiv 2 z$, $T_{2}(z) \equiv 4 z^{2}-2, T_{3}(z) \equiv 8 z^{3}-6 z, \ldots$ which are mutually orthogonal on the interval $-1 \leqq z \leqq 1$ with respect to the norm function $\left(1-z^{2}\right)^{-1 / 2}$ and are also orthogonal* on $\gamma$ and on every ellipse confocal with $\gamma$ with respect to the norm function $\left|1-z^{2}\right|^{-1 / 2}$.

As in the proof of Theorem 2, we have, for $z$ on and within $\gamma$, the relation

$$
\left|f(z)-\sum_{k=0}^{n} c_{k} T_{k}(z)\right| \leqq\left(M^{\prime} \log n\right) / n^{p+\alpha},
$$

where $M^{\prime}$ is independent of $n$ and $z$. The expansion $\sum_{k=0}^{\infty} c_{k} T_{k}(z)$ of $f(z)$ on $\gamma$ converges uniformly to $f(z)$ on $\gamma$ and hence is an expansion of the usual form in terms of orthogonal polynomials:

$$
c_{k} \int_{\gamma}\left|T_{k}(z)\right|^{2} \cdot\left|1-z^{2}\right|^{-1 / 2} \cdot|d z|=\int_{\gamma} f(z) \bar{T}_{k}(z)\left|1-z^{2}\right|^{-1 / 2} \cdot|d z| .
$$

Results analogous to Theorems 3 and 4 for the case that $f(z)$ is uniformly bounded interior to $\gamma$ or is analytic interior to $\gamma$ with poles or certain other singularities on $\gamma$ are due to Bernstein $\dagger$ and to de la Vallée Poussin (op. cit., chaps. 8 and 9).

4. Approximation to a periodic function on the axis of reals. A conformal transformation different from that used in Theorems 3 and 4 will now give further results from Theorems 1 and 2 .

THEOREM 5. Let the function $f(z)$ be periodic with period $2 \pi$, and let there exist trigonometric polynomials $t_{n}(z)$ of respective degrees $n$ such that we have for all real $z=x+i y$

$$
\left|f(z)-t_{n}(z)\right| \leqq M / n^{p+\alpha+1} \rho^{n}, \quad 0<\alpha \leqq 1, \rho>1,
$$

where $p$ is a non-negative integer. Then the function $f(z)$ can be analytically extended so that it is analytic throughout the band $|y|<\log \rho$, is

* Walsh, this Bulletin, vol. 40 (1934), pp. 84-88.

$\dagger$ †eçons sur les Propriêtés Extrémales, Paris, 1926, chap. 3. 
continuous in the corresponding closed region, and on the lines $y= \pm \log \rho$ satisfies condition (8).

The transformation $w=e^{i z}$ carries the line $y=0$ into the unit circle $|w|=1$, the band $|y|<\log \rho$ into the annulus $\rho>|w|>1 / \rho$, the function $f(z)$ into a function analytic and single-valued in that annulus, and the trigonometric polynomial $t_{n}(z)$ on $y=0$ into the trigonometric polynomial $t_{n}(\theta)$ on $|w|=1$ with $w=e^{i \theta}$. The hypothesis of Theorem 1 is satisfied, and an inequality of form (3) on the circles $|w|=\rho$ and $|w|=1 / \rho$ leads to the conclusion of Theorem 5 .

The given sequence $t_{n}(z)$ can be expressed on $y=0$ as a sequence of polynomials in $w=e^{i z}$ and $1 / w$, with $z=x=\theta$ :

$$
\begin{aligned}
& \sin k z=-\frac{i}{2}\left(e^{i k z}-e^{-i k z}\right)=-\frac{i}{2}\left(w^{k}-w^{-k}\right), \\
& \cos k z=\frac{1}{2}\left(e^{i k z}+e^{-i k z}\right)=\frac{1}{2}\left(w^{k}+w^{-k}\right) .
\end{aligned}
$$

These equations are then valid even if $y$ is different from zero.

It follows from the proof of Theorem 1 that the sequence $t_{n}(z)$, expressed in trigonometric form, converges in the closed region $|y| \leqq \log \rho$, with an error not greater than $M_{1} / n^{p+\alpha}$.

THEOREM 6. Let the function $f(z)$ be periodic with period $2 \pi$, let $f(z)$ be analytic in the band $|y|<\log \rho$, where $z=x+i y$, and continuous in the corresponding closed region, and let $f^{(p)}(z), p$ a non-negative integer, satisfy a Lipschitz condition of order $\alpha,(0<\alpha \leqq 1)$, on the lines $y= \pm \log \rho$. Then there exist trigonometric polynomials $t_{n}(z)$ of respective degrees $n$ such that we have for all real $z$

$$
\left|f(z)-t_{n}(z)\right| \leqq M / n^{p+\alpha} \rho^{n},
$$

where $M$ is a constant independent of $n$ and $z$.

The detailed proof of Theorem 6 is readily supplied by the reader by use of the same transformation $w=e^{i z}$ and the method of proof of Theorem 4 . It is of interest to note that the function $t_{n}(z)$ appears also for complex values of $z$ as the sum of the first $n+1$ terms of a series of the form

$$
\frac{1}{2} a_{0}+\sum_{k=1}^{\infty}\left(a_{k} \cos k z+b_{k} \sin k z\right) \text {. }
$$

As in Theorem 2, we have

$$
\left|f(z)-t_{n}(z)\right| \leqq\left(M^{\prime} \log n\right) / n^{p+\alpha}, \quad \text { for }|y| \leqq \log \rho,
$$

where $M^{\prime}$ is independent of $n$ and $z$. 
From the orthogonality of the functions $w^{k}$ on $|w|=\rho$ it follows that the transformed functions $e^{i k z}, k=\cdots,-1,0,1,2, \cdots$, form an orthogonal set on the line segment $y=-\log \rho,(0 \leqq x \leqq 2 \pi)$, with respect to the norm function $|d w / d z|=\left|d e^{i z} / d z\right|=e^{-y}$. This norm function is a nonvanishing constant on the segment and may therefore be omitted. Thus

$$
\int_{-i \log \rho}^{2 \pi-i \log \rho} e^{i k z} \cdot \overline{e^{i l z}} \cdot d x=0, \quad k \neq l,
$$

where $k$ and $l$ are integers, positive, negative, or zero. Of course this orthogonality condition holds on any interval $x_{0} \leqq x \leqq x_{0}+2 \pi, y=y_{0}$, the respective limits of integration being $x_{0}+i y_{0}$ and $x_{0}+2 \pi+i y_{0}$.

The set $e^{i k z}$ is closed (with respect to the class of continuous functions) on the interval $x_{0} \leqq x \leqq x_{0}+2 \pi, y=y_{0}$, as is seen by transformation to the $w$-plane. On every interval $x_{0} \leqq x \leqq x_{0}+2 \pi, y=y_{0}$, $\left|y_{0}\right| \leqq \log \rho$, the sequence

$$
\begin{aligned}
t_{n}(z) & =\frac{1}{2} a_{0}+\sum_{k=1}^{n}\left(a_{k} \cos k z+b_{k} \sin k z\right)=\sum_{k=-n}^{n} c_{k} e^{i k z}, \\
2 c_{k} & =a_{k}-i b_{k}, \quad 2 c_{-k}=a_{k}+i b_{k},
\end{aligned}
$$

is the sum of the first $2 n+1$ terms of the uniformly convergent formal development on that interval of the function $f(z)$ in terms of the orthogonal functions $e^{i k z}$, so that the coefficients are given by formulas of the usual type:

$$
c_{k}=\frac{e^{2 k y_{0}}}{2 \pi} \int_{x_{0}+i y_{0}}^{x_{0}+2 \pi+i y_{0}} \overline{e^{i k z}} \cdot f(z) \cdot d z,
$$

where on $y=y_{0}$ we have $\overline{e^{i k z}}=e^{-i k \bar{z}}=e^{-k y_{0}}(\cos k x-i \sin k x)$.

Analogs of Theorems 5 and 6 have been established by de la Vallée Poussin (op. cit., chaps. 8 and 9) for the case that $f(z)$ is bounded for $|y|<\log \rho$ or has poles or other singularities on the lines $y= \pm \log \rho$.

There is an obvious discrepancy of unity in the exponents of $n$ that appear in Theorems 1 and 2, in Theorems 3 and 4, and in Theorems 5 and 6. This discrepancy is inherent in the nature of the problem, as is shown by examples that the writers will publish on another occasion.

HARVARD UNIVERSITY AND

Georgia School of Technology 